\title{
El cáncer en el cine. Un recurso para los profesionales de la salud
}

M.T. Icart-Iserna , M.R. Rozas-García a , V. Sanfeliu-Cortes b, H. Viñas-Llebota , M.P. Fernández-Ortega c, M.C. Icart ${ }^{d}$

Objetivo. Describir las características de los pacientes y del proceso oncológico, a través de una muestra de películas disponibles en DVD. Materiales y métodos. Estudio descriptivo transversal. Se analizó una muestra de conveniencia de películas en las que el cáncer tenía carácter'puntual', 'relevante' o 'argumental'. Cada película fue visionada por dos observadores que registraron variables del paciente (edad, sexo, estado civil, etc.), del proceso oncológico (síntomas, pruebas diagnósticas, tratamientos y evolución) y del entorno sanitario, entre otras. Resultados. Se analizaron 33 películas producidas en 11 países (1939-2008). El $58 \%$ de los pacientes eran varones; y el $47 \%$ pertenecía a las clases altas y media-alta. La localización más frecuente del cáncer fue en cerebro y en sangre, con seis casos para cada localización. La media de síntomas por título fue de 3,63; la clínica general (dolor, fatiga, anorexia, etc.) se observó en el 43,32\% de las películas. Las pruebas diagnósticas se mencionaron en el $85 \%$ de los títulos. El tratamiento más frecuente fue la analgesia y la quimioterapia; no obstante, fallecieron 19 pacientes (58\%). Los médicos y las enfermeras intervinieron en 28 y 22 títulos, respectivamente. Conclusiones. El cáncer que muestra el cine difiere de la realidad: por cuanto el cine prefiere los pacientes jóvenes, de clase social alta y las localizaciones más fotogénicas. La clínica, las pruebas diagnósticas y los tratamientos suelen reflejar la realidad, sobre todo en las películas de las últimas décadas y en las que el cáncer tiene carácter 'argumental'; algunos de cuyos títulos pueden ser un recurso de primer orden para la formación de los profesionales de la salud.

Palabras clave. Cáncer. Cine. Proceso oncológico. Pruebas diagnósticas. Tratamientos.
Cancer in the movies. A resource for health professionals

Aim. To describe the characteristics of the patients and the cancer process, through a sample of films available in DVD. Materials and methods. Cross-sectional descriptive study. A sample of convenience of films was analyzed in which the cancer had 'prompt', 'relevant' or 'plot' character. Each film was viewed by two observers who recorded patient variables (age, sex, marital status, etc.), the cancer process (symptoms, diagnostic tests, treatments and evolution) and the health care environment, among others. Results. 33 films produced by 11 countries (1939-2008) were analyzed. 58\% of patients were male and $47 \%$ belonged to the upper and upper middle social classes. The most common site for the cancer was the brain and blood, with six cases in each location. The symptoms average was 3.63 per film; the general symptoms (pain, fatigue, anorexia, etc.) were considered in $43.32 \%$ of the films. Diagnostic tests were mentioned in $85 \%$ of movies. The most frequent treatment was chemotherapy and analgesia, however death hits 19 (58\%) of the patients. Doctors and nurses turn up in 28 and 22 films titles respectively. Conclusions. The cancer showed by films is different from reality: movies prefer younger patients, higher social class and the most photogenic cancer's locations. The symptoms, diagnostic tests and treatments tend to reflect reality, especially in 'plot' movies produced in the last decades. Some of those films may be a first hand resource for training health professionals.

Key words. Cancer. Cancer process. Diagnostic. Films. Treatments. a Departamento de Enfermería de Salud Pública, Salud Mental y Materno-Infantil.

b Departamento de Enfermería Fundamental y Médico-Quirúrgica. Escuela de Enfermería. Universitat de Barcelona. Institut Català d'Oncologia.

d Área Básica de Salud Sant Josep. Institut Català de la Salut. L'Hospitalet de Llobregat, Barcelona, España.

Correspondencia Dra. M. Teresa Icart Isern. Escuela de Enfermería. Universitat de Barcelona. Campus Bellvitge. Feixa Llarga, s/n. E-08907 L'Hospitalet de Llobregat (Barcelona)

Fax

+34 934024297

E-mail

mticart@ub.edu

Financiado por la Universitat de Barcelona, Ajuts a la Recerca del Campus de Bellvitge (ACESB07/04).

\section{Introducción}

La utilización del cine por parte de los profesionales de la salud es un reconocimiento de las posi- 
bilidades que ofrece el séptimo arte como recurso para: el aprendizaje de actitudes en la atención a los enfermos [1], la revisión de diferentes patologías, la identificación y valoración de pruebas diagnósticas y tratamientos [2,3], el análisis de las relaciones entre pacientes y profesionales [4], la identificación de las diferencias socioculturales en la vivencia de la enfermedad, etc. [5].

En algunas películas, las enfermedades, entre ellas el cáncer, se utilizan de modo contextual, para dar verosimilitud al argumento; en otras, son el centro del argumento. Según García-Sánchez [2], las películas se pueden clasificar en: 'saludables' (no hay rastro de enfermedad), 'puntuales' (sólo aparece alguna alusión a la enfermedad), 'relevantes o de papel importante' (el proceso mórbido acompaña de modo constante a alguno/s de los protagonistas) y 'argumentales' (la película se centra en el impacto que causa la enfermedad).

Concretamente, el abordaje cinematográfico del los procesos oncológicos ha sido objeto de destacados artículos publicados en la Revista de Medicina y Cine/Journal of Medicine and Movies de la Universidad de Salamanca [6-8]. No obstante, en la bibliografía revisada no se ha encontrado ningún estudio descriptivo de los pacientes oncológicos. Este tipo de estudio permitiría disponer de un material docente útil para la formación de futuros profesionales de la salud [9], pero también podría emplearse en asociaciones de pacientes y familiares como eje de discusión para un mayor conocimiento de la enfermedad y sus repercusiones familiares y sociales [10].

El objetivo de este estudio fue describir cómo el cine presenta el cáncer a través de una muestra de películas disponibles en DVD. Los objetivos específicos fueron: describir las variables sociodemográficas de los pacientes y su proceso neoplásico (clínica, pruebas diagnósticas, tratamiento y evolución) así como las características del entorno sociofamiliar y sanitario en el que se desarrollaba la enfermedad.

\section{Materiales y métodos}

Se trata de un estudio descriptivo cuyas unidades de análisis fueron películas en las que uno de los personajes presentaba cáncer. Se incluyeron películas, que en el periodo del estudio, marzo de 2008 a enero de 2009, estuvieran disponibles en DVD y contaran con una versión en español (original, doblada o subtitulada); se trata de una muestra no probabilística, de conveniencia. Para la identificación de las películas se consultó la clasificación de la web de Salud y Cine [11] y el Diccionario temático de cine [12].

Las películas se clasificaron según la importancia que tiene el cáncer en el guión (narración de la acción dividida en secuencias) en puntuales, relevantes y argumentales. Se consideraron puntuales aquéllas que ofrecían al menos tres secuencias sobre una manifestación clínica, prueba diagnóstica o tratamiento asociados al cáncer, que afectaba a alguno de los personajes, aunque no fuera el protagonista. Las películas relevantes fueron aquéllas en las que la/el protagonista principal tenía cáncer, del que se describían manifestaciones clínicas, se practicaba alguna prueba diagnóstica y/o se aplicaba algún tratamiento; estas situaciones se producían en al menos cuatro secuencias cinematográficas. Las argumentales eran aquellas que relataban la enfermedad a través de un texto narrativo o discurso en el que el/la protagonista presentaba un diagnóstico de cáncer; se describían las fases del proceso (clínica, diagnóstico, tratamiento y resolución) que se transmitía tanto en el guión literario (historia), como en el guión técnico (encuadres, iluminación, movimiento de la cámara, música, efectos sonoros, etc.).

Cada película fue visionada por dos miembros del equipo, que registraron las variables a estudiar en una ficha elaborada ad hoc. En caso de desacuerdo, un tercer miembro del equipo analizaba la secuencia para llegar a un consenso.

La información recogida comprendió una serie de variables sociodemográficas del paciente (sexo, edad, estado civil, ocupación, etc.) y otras específicas del proceso oncológico (localización del tumor, clínica, pruebas diagnósticas, tratamiento y evolución). También se registró la intervención de profesionales de la salud (médicos, enfermeras, psicólogos, etc.) y los espacios en que el paciente recibía atención (hospital, consultorio, centro socio-sanitario, hogar, etc.). En los títulos de crédito se identificó la asesoría científica y/o los agradecimientos a profesionales o instituciones sanitarias.

En las películas donde el cáncer tenía carácter puntual o relevante se registró el número de secuencias que abordaban el proceso oncológico; en las que tenía carácter 'argumental' no se consignó esta variable por entender que el cáncer era omnipresente. 
Tabla I. Títulos de las películas estudiadas, localización del cáncer y su carácter en la película.

\begin{tabular}{|c|c|c|c|}
\hline Titulo de la película & Director, año & Localización del cáncer & $\begin{array}{l}\text { Carácter del cáncer } \\
\text { en la película }\end{array}$ \\
\hline Ahora onunca & R. Reiner, 2007 & Cerebro & Relevante \\
\hline Amar la vida & M. Nichols, 2001 & Ovario & Argumental \\
\hline Amarga victoria & E. Goulding, 1939 & Cerebral & Argumental \\
\hline Caro diario & N. Moretti, 1993 & Pulmón & Relevante \\
\hline El día de mañana & R. Emmerich, 2003 & Leucemia & Puntual \\
\hline Eldoctor & R. Haines. 1991 & Laringe & Argumental \\
\hline El juego de la verdad & A. Fernández, 2004 & Cerebral & Relevante \\
\hline El llanto de la mariposa & F. Strecker, 2003 & Leucemia & Relevante \\
\hline El tiempo que queda & F. Ozon, 2005 & Hígado. Metástasis & Argumental \\
\hline Gritos y susurros & I. Bergman, 1978 & Útero & Argumental \\
\hline La caja china & W. Wang, 1997 & Leucemia & Puntual \\
\hline La fuerza del cariño & J. Brooks, 2001 & Mama & Relevante \\
\hline La suerte de Emma & S. Taddicken, 2007 & Páncreas & Relevante \\
\hline La noche de las chicas & N. Hurran, 1998 & Mama. Metástasis & Relevante \\
\hline La puta y la ballena & L. Puenzo, 2004 & Mama & Relevante \\
\hline Lavida & J.P. Amèris, 2001 & Estómago. Metástasis & Argumental \\
\hline Las chicas del calendario & N. Cole, 2003 & Leucemia & Puntual \\
\hline Las invasiones bárbaras & D. Arcand, 2003 & Hígado & Argumental \\
\hline Love story & A. Hiller, 1970 & Leucemia & Relevante \\
\hline Magnolia & P.T. Anderson, 1999 & Cerebro. Pulmón & Puntual \\
\hline Mi vida & B.J. Rubin, 1993 & Renal & Argumental \\
\hline Mi vida sin mí & I. Coixet, 2002 & Ovario. Metástasis & Argumental \\
\hline Noviembre dulce & P. O'Connor, 2001 & Linfoma & Relevante \\
\hline Obsesión & D. Sirk, 1954 & Cerebro & Relevante \\
\hline Otoño en Nueva York & J. Chen, 2000 & Neuroblastoma & Relevante \\
\hline Peligro inminente & P. Noyce, 1994 & Páncreas & Puntual \\
\hline Phenomenon & J. Tuteltaub, 1996 & Cerebro & Relevante \\
\hline Planta $4 .^{a}$ & A. Mercero, 2003 & Osteosarcoma & Argumental \\
\hline Presidente Miterrand & R. Guédiguian, 2005 & Próstata & Puntual \\
\hline Quédate a mi lado & C. Columbus, 1998 & Tórax & Relevante \\
\hline Te estoy perdiendo & D. Cronenberg, 2002 & Pulmón & Puntual \\
\hline Tierras de penumbra & R. Attenborough, 1993 & Óseo & Relevante \\
\hline Venus & R. Michell, 2006 & Próstata & Puntual \\
\hline
\end{tabular}


Para el análisis se aplicaron estadísticos descriptivos y se utilizó la aplicación SPSS v. 16.0.

\section{Resultados}

Se analizaron 33 películas realizadas entre 1939 y 2008 (20 posteriores a 2000). El cáncer tiene carácter 'argumental' en 10 títulos, 'relevante' en 15 y en el resto aparece de forma 'puntual' (Tabla I).

Estados Unidos produjo 17 títulos, le sigue España con 5, Francia con 5, Gran Bretaña con 3, Canadá y Alemania con 2 cada uno y, finalmente, Argentina, Italia, Japón, México y Suecia con una película cada país. Seis títulos fueron coproducidos por dos países.

En cuanto a las variables sociodemográficas, se observa que el cáncer afecta principalmente al sexo masculino (razón de masculinidad de 1,35). La edad con mayor número de personas con cáncer se sitúa entre los 40 y 45 años, oscilando entre los 8 y los 70 años, aproximadamente. La distribución del resto de variables sociodemográficas: estado civil, profesión y clase social, se presenta en la tabla II. Todos los pacientes pertenecen a la raza blanca salvo un coprotagonista, también con cáncer, que es de raza negra (Ahora o nunca).

El cáncer localizado en el cerebro y en sangre, con seis casos cada uno, ocupa los dos primeros lugares entre las películas visionadas. El cáncer ginecológico (ovario, endometrio y cérvix), al igual que el de mama, aparecen en tres títulos cada uno (Fig. 1); en cinco títulos se menciona la presencia de metástasis.

Todas las películas presentan alguna secuencia con síntomas o signos derivados del propio cáncer o como consecuencia del tratamiento (Tabla III) con una media de 3,63 síntomas por título. Los síntomas y signos de tipo general o sistémico son los más habituales $(43,32 \%)$, seguidos por los trastornos gastrointestinales $(16,67 \%)$ casi siempre asociados a efectos secundarios de los tratamientos.

En 28 películas (85\%) se menciona alguna prueba diagnóstica, siendo la analítica en sangre la más citada (10 títulos), le sigue la tomografía axial computarizada (Fig. 2). En cinco y siete películas se practican dos y tres pruebas respectivamente y en seis títulos se especifica que se va a proceder a un chequeo o exploración que incluye varias pruebas.

\begin{tabular}{|c|c|c|}
\hline \multicolumn{3}{|c|}{$\begin{array}{l}\text { Tabla II. Variables sociodemográficas de } \\
\text { los protagonistas que presentan cáncer } \\
\text { en los } 33 \text { títulos analizados. }\end{array}$} \\
\hline & $n$ & $\%$ \\
\hline \multicolumn{3}{|l|}{ Sexo } \\
\hline Hombres & 19 & 58 \\
\hline Mujeres & 14 & 42 \\
\hline \multicolumn{3}{|l|}{ Estado civil } \\
\hline Solteros & 11 & 33 \\
\hline Casados & 12 & 37 \\
\hline En pareja & 2 & 6 \\
\hline Divorciados/separados & 5 & 15 \\
\hline Viudos & 3 & 9 \\
\hline \multicolumn{3}{|l|}{ Profesión } \\
\hline Ama de casa & 6 & 18 \\
\hline Estudiante & 6 & 18 \\
\hline Medios de comunicación & 3 & 9 \\
\hline Empresarios & 2 & 6 \\
\hline Profesores & 2 & 6 \\
\hline Otras & 14 & 43 \\
\hline \multicolumn{3}{|l|}{ Clase social } \\
\hline Alta & 6 & 18 \\
\hline Media-alta & 10 & 30 \\
\hline Media & 12 & 37 \\
\hline Media-baja & 5 & 15 \\
\hline
\end{tabular}

En 28 títulos se aplica algún tratamiento, siendo el más habitual la analgesia, seguida de la quimio- 


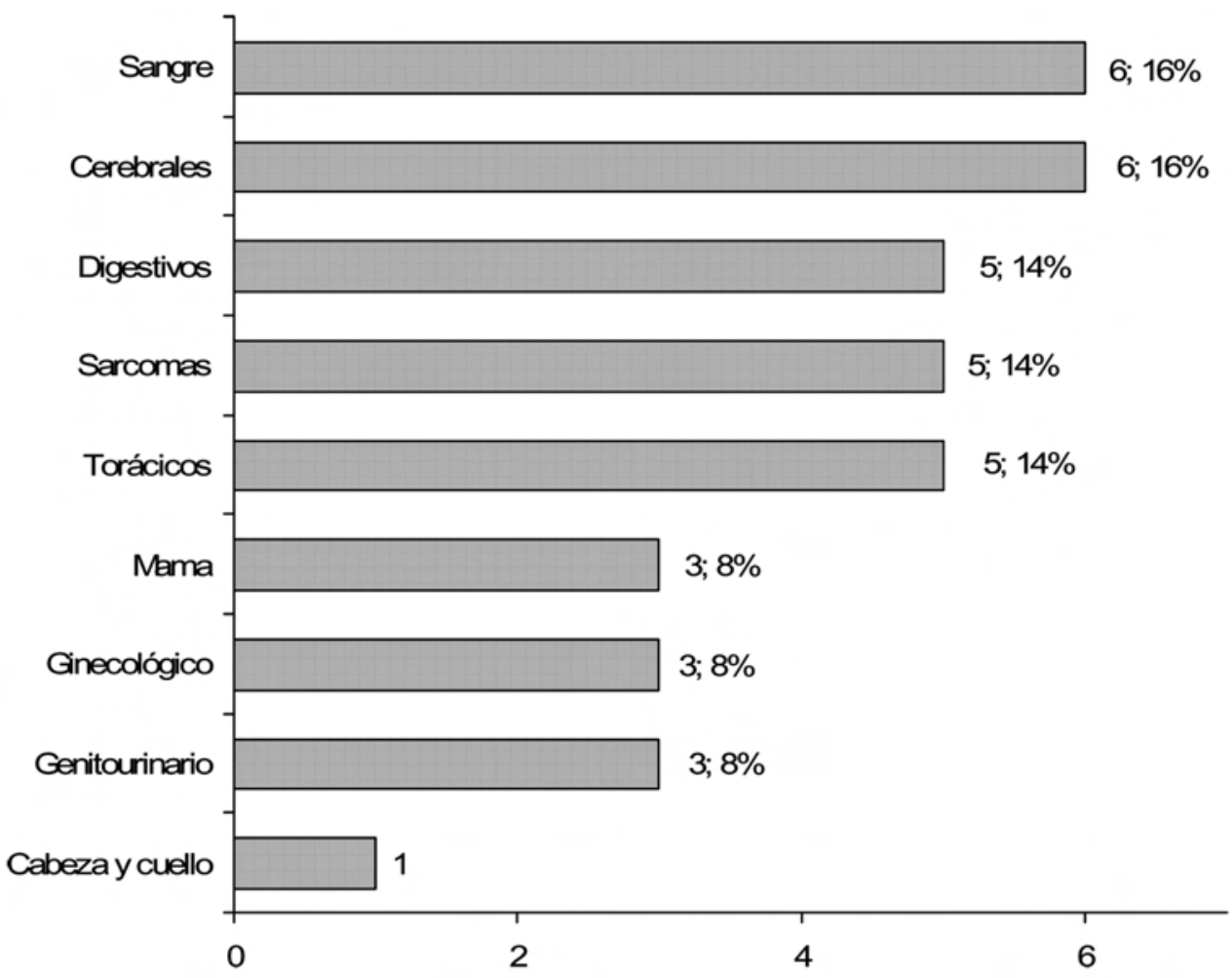

Figura 1. Localización del cáncer en los 33 títulos de las películas analizadas (cifras absolutas y porcentajes).

terapia (Fig. 3). En ocho y seis títulos se emplean dos y tres tratamientos, respectivamente. En Caro diario, se llegan a aplicar seis tratamientos (reflexoterapia, baños, acupuntura, relajación, etc.) para el mismo paciente. El enfermo con cáncer muere en 19 películas, empeora en seis, se cura en tres y se desconoce el desenlace en cinco títulos.

Entre los profesionales de la salud, los médicos y las enfermeras aparecen en 28 y 22 títulos, respectivamente. Otros profesionales (psicólogos, fisioterapeutas, etc.) intervienen en seis películas y en otras tantas se incluye la figura de un/a religioso/a. Las instalaciones hospitalarias aparecen en escenas de 27 películas y las consultas externas en 10 casos. El sistema de salud es de tipo privado en 13 títulos, público en seis, mixto en cuatro y no se puede determinar en 10 películas. Junto al enfermo de cáncer aparecen otros enfermos en 14 títulos, algunos de los cuales también sufren cáncer.

La media de secuencias relacionadas con el cáncer fue de seis para las que el cáncer tenía carácter relevante y 2,5 para las puntuales.
En los títulos de crédito se agradece la colaboración de alguna institución sanitaria o el asesoramiento de algún profesional en 15 títulos; esta práctica es más frecuente en las últimas décadas.

\section{Discusión}

En las películas, el intervalo de edad más frecuente entre los pacientes (entre los 40 y 45 años) es inferior al que se suele diagnosticar el cáncer (entre 55 y 64 años) [13]. El predominio de la juventud y la clase social media-alta muestra cómo las preferencias del séptimo arte pueden distorsionar la realidad, esta práctica también se observa en análisis de otros estudios $[4,6]$.

Mientras el cáncer localizado en el cerebro y en la sangre ocupa los dos primeros lugares en el cine, en la realidad, esta posición la ocupan el cáncer de pulmón, en el hombre, y el de mama, en la mujer. Al igual que en el trabajo de Lederer [14], se observa que los productores prefieren los tipos de cán- 
cer menos dramáticos y los que causan un menor impacto visual o son más fotogénicos. En ese sentido, no aparece ningún cáncer colorrectal, aunque su incidencia ocupa el segundo lugar en hombre y mujeres adultos [13]; tampoco aparecen tumores de la cavidad bucal ni cáncer cutáneo $[4,14]$. En cuanto a la localización del cáncer según la edad, el cine permanece fiel a la realidad, y presenta el cáncer óseo y la leucemia en menores de 25 años, mientras que el cáncer ginecológico y el de próstata lo reserva para protagonistas adultos [15].

Los síntomas generales, como el dolor y la fatiga, ocupan los primeros lugares en los títulos analizados, siendo las películas donde el cáncer tiene carácter 'argumental' (Wit, Mi vida sin mí, El doctor, etc.) las que describen con mayor fidelidad los síntomas y signos. En la realidad, los síntomas generales (dolor, fatiga, anorexia, pérdida de peso, etc.), asociados a la enfermedad, a los tratamientos o a los factores psicosociales y emocionales, ocurren en el $85 \%$ de los pacientes en fases avanzadas del proceso [16].

En cuanto a las pruebas diagnósticas se observa, al igual que en otros estudios $[4,7,14]$, que las películas realizadas en la última década incorporan los avances tecnológicos (Camino, La puta y la ballena, Mi vida sin mí) casi ausentes en las producciones anteriores (Amarga victoria, Love story, Gritos y susurros).

Las circunstancias en que se produce la muerte del paciente (58\% de la muestra) son similares a las descritas en la obra de Reagan et al [17]. La mortalidad por cáncer en el cine describe una escena más pesimista que la correspondiente al estudio Eurocare-4 [18], realizado en 23 países europeos y que señala una supervivencia del $59 \%$ y del $49,5 \%$ para mujeres y hombres, respectivamente.

La imagen que el cine transmite de los profesionales es variada, como también lo es su grado de implicación en el proceso oncológico. Se han encontrado similitudes con otros autores $[4,19]$ en cuanto a la actuación, a veces compasiva y empática y otras egoísta e interesada, por parte de los profesionales de la medicina y la enfermería, que son mayoría frente a otros colectivos. Los títulos analizados confirman al hospital como el primer espacio donde se producen las actuaciones sanitarias en el proceso oncológico.

Entre las limitaciones del estudio destacan la dificultad en la identificación de películas donde aparezca el cáncer o su inexistencia en DVD
Tabla III. Clínica observada en los títulos analizados.

\begin{tabular}{|c|c|c|}
\hline & $n$ & $\%$ \\
\hline General o sistémico & 52 & 43,32 \\
\hline Dolor & 16 & 13,33 \\
\hline Fatiga & 16 & 13,33 \\
\hline Lipotimia & 9 & 7,50 \\
\hline Pérdida de peso & 6 & 5,00 \\
\hline Otros: hemoptisis, escalofrío, etc. & 5 & 4,16 \\
\hline Gastrointestinal & 20 & 16,67 \\
\hline Vómitos & 8 & 6,67 \\
\hline Náuseas & 4 & 3,33 \\
\hline Úlceras bucales & 3 & 2,50 \\
\hline Otros: diarrea, disfagia, etc. & 5 & 4,17 \\
\hline $\begin{array}{l}\text { Sistema nervioso central } \\
\text { o neurológico }\end{array}$ & 18 & 15,00 \\
\hline Alteración de la visión & 4 & 3,33 \\
\hline Cefalea & 3 & 2,50 \\
\hline Insomnio & 3 & 2,50 \\
\hline $\begin{array}{l}\text { Otros: alteraciones de la marcha, } \\
\text { hormigueo, pérdida de fuerza, } \\
\text { alteraciones de la sensibilidad, etc. }\end{array}$ & 8 & 6,67 \\
\hline Respiratorio & 17 & 14,16 \\
\hline Tos & 7 & 5,83 \\
\hline Disnea & 7 & 5,83 \\
\hline Otros & 3 & 2,50 \\
\hline Piel y tegumentos & 11 & 9,17 \\
\hline Alopecia & 8 & 6,67 \\
\hline Prurito & 3 & 2,50 \\
\hline Inflamación ganglionar & 2 & 1,68 \\
\hline
\end{tabular}




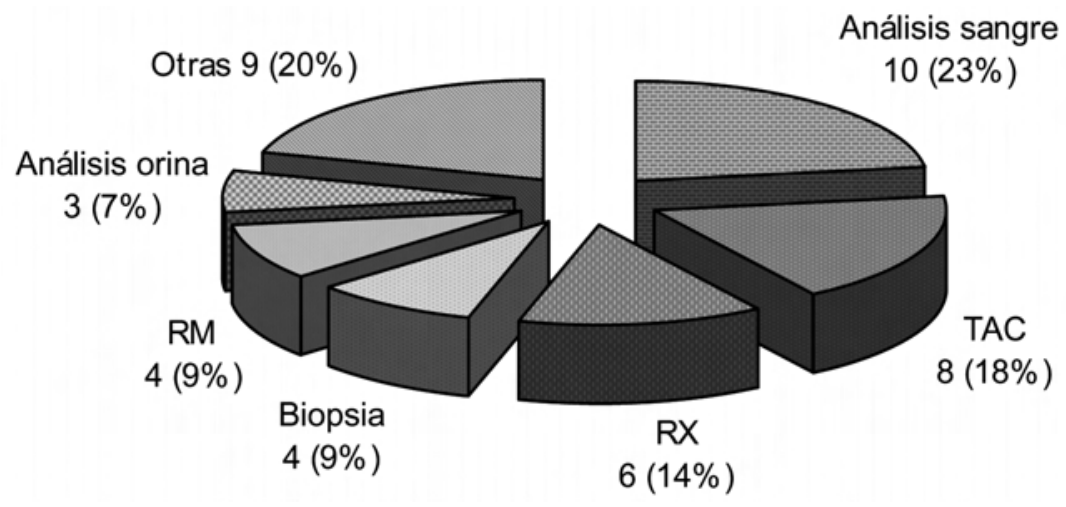

Figura 2. Pruebas diagnósticas identificadas en los 33 títulos de las películas analizadas (cifras absolutas y porcentajes). Otras: ecografía, tomografía por emisión de positrones, escáner, marcadores tumorales, etc. RM: resonancia magnética; TAC: tomografía axial computarizada; RX: radiografía.

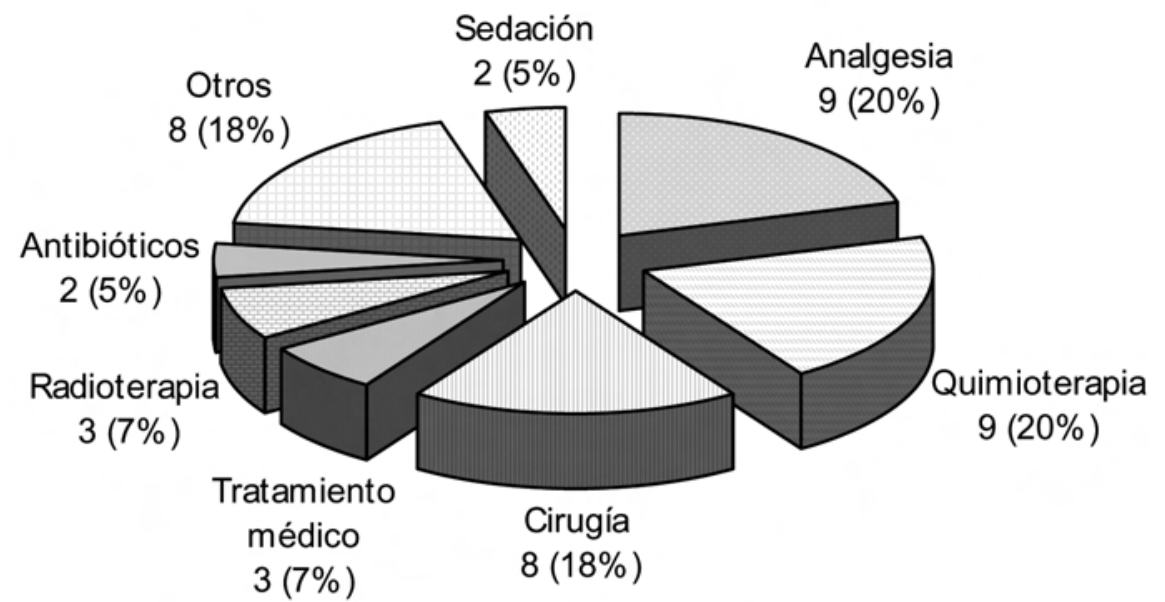

Figura 3. Tratamientos que se mencionan en los 33 títulos de las películas analizadas (cifras absolutas y porcentajes). Otros: antihistamínicos, antieméticos, baños, vahos, trasplante de médula ósea, transfusión.

(bien porque no se han editado en ese formato o por estar descatalogados en el periodo de la realización de este estudio). En ese sentido, títulos como Lluvia negra (S. Imamura, 1989), Mi padre (E.D. Goldberg, 1989), La habitación de Marvin (J. Zaks, 1997) o Agnes Brown (A. Houston, 1999), entre otros, se podría añadir a la lista de películas sobre cáncer. Aún así la muestra difícilmente podría ser probabilística debido a la extraordinaria y constante producción cinematográfica.

Otra limitación reside en la valoración de variables como la edad o la clase social, como también de algunos síntomas que se podían confun- dir (fatiga con disnea, fatiga con dolor), ya que no siempre se explicitaban en los diálogos. Parte de este problema se corrigió con el doble visionado y la opinión, en caso de duda, de una tercera persona. Otra posible fuente de error se puede deber a la falta de fidelidad de la traducción de la versión original al castellano, idioma en el que se realizó el visionado. Cabe destacar que este trabajo se ha limitado a analizar aspectos concretos del proceso oncológico, pero sería de gran interés estudiar las emociones, sentimientos, creencias y valores que manifiesta el paciente y quienes le acompañan o asisten durante la enfermedad. 
Entre las implicaciones prácticas de este estudio destacamos el posible uso de las películas visionadas para la formación de futuros profesionales de la salud. Cabe señalar que el carácter formativo de una película tiene que ver con su argumento pero, sobre todo, se lo confiere la intencionalidad del docente que la utiliza para promover la reflexión y el análisis de un problema de salud. Por esta razón, el uso del cine como recurso didáctico se inscribe en un proceso dinámico que debe contar con la complicidad del docente y del alumno $[8,9,20]$.

\section{Bibliografía}

1. González-Blasco P, Roncoletta AFT, Moreto G, Levites MR, Janaudis MA. Medicina de familia y cine: un recurso humanístico para educar la afectividad. Aten Primaria 2005; 36: 566-72.

2. García-Sánchez JE, Frenadillo MJ, García-Sánchez E. El cine en la docencia de las enfermedades infecciosas y la microbiología clínica. Enferm Infecc Microbiol Clin 2002; 20: 403-6.

3. Alexander M, May MN, Pettice YJ. Cinemeducation: an innovative approach to teaching psychosocial medical care. Fam Med 1994; 26: 430-3.

4. Raj YP. Medicine, myths, and the movies. Hollywood's misleading depictions affect physicians, patients alike. Postgrad Med 2003; 113: 9-10, 13.

5. Álvarez D, Cariacedo JJ, Gómez JM, López S, Peña JI, Pereda FJ, et al. El valor de la diversidad. El cine como recurso didáctico en la educación intercultural. Madrid: Carprinter; 2003.
6. Aldarondo R. El cine como alivio y como objetivo final. Rev Med Cine 2008: 4: 108-12.

7. Ortega MJ, Rodríguez M, Sánchez M, Bravo E, Corredera A, Ojeda MA, et al. Aprendiendo con el cine: un instrumento puente entre la realidad y las ideas en el proceso de morir. Rev Med Cine 2008; 4: 113-21.

8. Astudillo W, Mendinueta C. El cine en la docencia de la medicina: cuidados paliativos y bioética. Rev Med Cine 2007; 3: 32-41

9. Icart MT. Metodología de la investigación y cine comercial: claves de una experiencia docente. Educ Med 2008; 11: 13-8.

10. Peske N, West B. Cineterapia avanzada. Barcelona: Ediciones B; 2003.

11. García E. La medicina en el cine. Salud y cine. URL: http://www.usal.es/ micromed.

12. Sánchez JL. Diccionario temático del cine. Madrid: Cátedra; 2004.

13. Sociedad Española de Oncología Médica. Cifras de cáncer en España 2009. URL: http://www.redaccionmedica. com/ redaccion/cancer_cifras_SEOM.pdf.

14. Lederer SE. Dark victory: cancer and popular Hollywood film. Bull Hist Med. 2007: 81: 94-115.

15. García E. Venus (2006) y el cáncer de próstata. Rev Med Cine 2007; 3: 172-3.

16. Gómez-Batiste X, Madrid F, Moreno F, Gracia A, Trelis J, Nabal M, et al. Breakthrough cancer pain: prevalence and characteristics in patients in Catalonia, Spain. J Pain Symptom Manage 2002; 24: 45-52.

17. Reagan LJ, Tomes N, Treicher PA. Medicine's moving pictures. New York: Boydell \& Brewer; 2007.

18. Verdecchia A, Francisci S, Brenner H, Gatta G, Micheli A, Mangone L, et al. Recent cancer survival in Europe: a 2000-02 period analysis of EUROCARE-4 data. Lancet Oncol 2007; 8: 784-96.

19. Dixon B. Scientists and their image. BMJ 1993; 307: 268.

20. Bosch F, Baños JE, Empleo de películas comerciales en la docencia de la farmacología. Educ Med 1999; 4: 197. 